\title{
Newly Isolated Paecilomyces lilacinus and Paecilomyces javanicus as Novel Biocontrol Agents for Plutella xylostella and Spodoptera litura
}

\section{Hoang Chinh NGUYEN ${ }^{1}$, Thi Van Anh TRAN ${ }^{1}$, Quoc Linh NGUYEN ${ }^{2}$, Nhu Nhut NGUYEN ${ }^{2}$, Minh Khiem NGUYEN ${ }^{1}$, Ngoc Thanh Tam NGUYEN ${ }^{1}$, Chia-Hung SU ${ }^{3 * *}$, Kuan-Hung LIN ${ }^{4 *}$}

\author{
${ }^{1}$ Ton Duc Thang University, Faculty of Applied Sciences, 700000 Ho Chi Minh City, Vietnam;nguyenhoangchinh@tdt.edu.vn; \\ bsy@staff.pccu.edu.tw;minhkhiem2710@gmail.com; theresapi@yahoo.com.tw \\ ${ }^{2}$ Gia Tuong Company Ltd., Binh Duong,Vietnam;nguyenquoclinh@giatuong.vn;nhunhutnguyen@yahoo.com.uk \\ ${ }^{3}$ Ming-Chi University of Technology, Graduate School of Biochemical Engineering, 24301 Taipei, \\ Taiwan; chsu@mail.mcut.edu.tw (**co-correspondingauthor) \\ ${ }^{4}$ Chinese Culture University, Graduate Institute of Biotechnology, 11114 Taipei, Taiwan; rlin@faculty.pccu.edu.tw (*orresponding author)
}

\begin{abstract}
Biocontrol offers an attractive alternative to the use of chemical pesticides in agricultural pest management. The development of high levels of resistance to chemical pesticides have forced researchers to find more alternative biological control agents. The aims of this study were to isolate Paecilomyces spp. with high virulence against diamondback moth (Plutella xylostella) and Oriental leafworm moth (Spodoptera litura), and to investigate suitable agro-industrial residues as a substrate used for solid state fermentation for sporulation of isolates. In this study, Paecilomyces spp. were isolated from soil and insects and identified by morphological and sequencing analyses. The pathogenicity of these isolates was evaluated on Pl. xylostella and $S$. litura to identify strains with the highest virulence. In addition, agro-industrial residues were used as a cheap substrate for investigating a suitable medium for sporulation on an industrial scale. Six strains of Paecilomyces spp. were isolated including one strain of $P$. lilacinus and five strain of $P$. javanicus. P. lilacinus PL01 showed the highest virulence against both Pl. xylostella and $S$. litura with respective $\mathrm{LT}_{50}$ values of 2.51 and 7.09 days. The five isolated $P$. javanicus strains also strongly infected $P l$. xylostella with $\mathrm{LT}_{50}$ values of 2.52 6.59 days. For sporulation, brown rice alone or brown rice mixed with rice husks and wheat bran or rice bran was suitable for cultivating these isolates. Two newly isolated species of Paecilomyces, P. lilacinus and $P$. javanicus, can be used as biological control agents for controlling Pl. xylostella and S. litura.
\end{abstract}

Keywords: agro-industrial residues, biocontrol, bio-pesticides, entomopathogenic fungi, solid-state fermentation, sporulation

\section{Introduction}

The diamondback moth (Plutella xylostella) and Oriental leafworm moth (Spodoptera litura) are major pests of cabbage, canola, broccoli, and other crucifers. To control these pests, farmers all over the world spend more than $\$ 1$ billion on chemical insecticides each year (Talekar and Shelton, 1993). However, many populations of these pests have become resistant to chemical pesticides and were detected in Asia (Syed, 1992), Central America (Perez and Shelton, 1997), the continental United States (Shelton and Wyman, 1992), and Hawaii (Tabashnik et al., 1990). Biocontrol offers an attractive alternative or supplement to the use of chemical pesticides in agricultural pest management to protect crops (Lopez et al., 2014; Kepenekci et al., 2015). The use of fungal agents for biological control of pests is an important strategy to minimize synthetic chemical pesticides which often have adverse effects on humans, animals, and the environment (Mar et al., 2012). However, it is hard to develop specific fungi as potential biopesticides because their sporulation on culture media usually depends on the species and the components used in the artificial media. There are several ways to manipulate fungi becoming a biocontrol agent, but fungi must be available in large quantities. In general, the success of microbial biopesticides depends on obtaining the pathogen at competitive prices to permit its mass-production and commercialization (Gao et al., 2007). Production processes for fungal biopesticides must be low-cost, and yield high concentrations of viable, virulent, persistent spores that can be stabilized to provide a product shelf-life of 12 18 months (Cliquet and Jackson, 2005).

The nutritional composition of the production medium significantly impacts the biocontrol efficacy, tolerance, desiccation, and persistence (Lane et al., 1991). Utilization of 
industrial residues or agricultural products is a great alternative to achieve a competitive price, resulting in the utilization of agro-industrial residues with added value (Soccol and Vandenberghe, 2003). Sorghum, white rice, wheat, coffee husks, rye, barley, sugarcane, cassava bagasse, refused potatoes, corn, beans, soy, glucose syrup, and grapes have been used as materials for spore production (Buzzini and Martini, 1999; Pandey et al., 2000; Kamp and Bidochka, 2002; Santa et al., 2005; Robl et al., 2009; Mishra and Thawani, 2016). Solidstate fermentation of these crop residues is advantageous because it is easy to carry out, and raw materials are cheap (Mishra and Thawani, 2016). In general, product costs and storage stability have driven the development of production and formulation processes (Ying and Feng, 2006).

The development of high levels of resistance to most chemical pesticides and the side effects of pesticides on natural enemies of the pest have forced researchers to find alternative biological control agents (Lacey et al., 1996; Lopez et al., 2014; Kepenekci et al., 2015). The genera Beauveria and Paecilomyces have been recognized as important biocontrol agents of aleyrodid pests of field and greenhouse crops (Wraight $e t a l$., 1998; Kepenekci et al., 2015; Ibrahim et al., 2016). Paecilomyces contains members which are often thermophilic, a perfect state for placement in the ascomycetous genera, Talaromyces and Thermoascus. Isarioidea contains mesophiles, including several well-known entomopathogenic or nematophagous species, such as Paecilomyces lilacinus and $P$. javanicus (Samson, 1974; Kepenekci et al., 2015). Paecilomyces lilacinus is a soil fungus and has been used as an efficient and common nematicide (Kiewnick and Sikora, 2006; Siddiqui and Futai, 2009; Kepenekci et al., 2015), and also as a controller of greenhouse insects and mite pests (Fiedler and Sosnowska, 2007; Wraigt et al., 2000; Zawadneak et al., 2015). To date, no comparison of the efficacy of different $P$. lilacinus and $P$. javanicus isolates against diamondback moth $(P l$. xylostella) and Oriental leafworm moth ( $S$. litura) has been reported. In this paper, we aimed to isolate new Paecilomyces spp. with high virulence against $P l$. xylostella and $S$. litura, and analyze the spore production of isolates in agro-industrial residues. The objectives of this study were to isolate and identify Paecilomyces spp. as biocontrol agents against diamondback moth (Plutella xylostella) and Oriental leafworm moth (Spodoptera litura) under laboratory conditions, and to investigate suitable agro-industrial residues as a substrate used for solid state fermentation for sporulation of isolates.

\section{Materials and Methods}

\section{Materials}

All the chemicals and reagents were purchased from SigmaAldrich Fine Chemicals (St. Louis, MO, USA). The cultural media were from Becton, Dickinson and Company (Franklin Lakes, NJ, USA). Primers were synthesized by Nam Khoa Biotek Comapany (Ho Chi Minh, Vietnam).

\section{Collection, isolation, and identification of Paecilomyces spp.}

Soils and naturally infected insects were collected from cultivated fields and greenhouse areas in Binh Duong Province, Vietnam. Dead insects with external signs of mycosis were collected and promptly transported to the laboratory. The fungi were isolated directly from dead insects by transferring external conidia from dead insects onto potato dextrose agar (PDA) medium amended with $0.01 \%(\mathrm{w} / \mathrm{w})$ chloramphenicol and $3 \%$ $(\mathrm{w} / \mathrm{w})$ sodium chloride plate $(\mathrm{PDA}+)$ and incubated at $28^{\circ} \mathrm{C}$ for 7 days (Pau et al., 2012). Two grams of soil were added to a flask containing $20 \mathrm{ml}$ sterilized water. The soil suspension was diluted into concentrations of $10^{-1}, 10^{-2}$, and $10^{-3}$ after shaking for $10 \mathrm{~min}$, subsequently spread onto PDA+ plates, and incubated at $28^{\circ} \mathrm{C}$ for 7 days (Pau et al., 2012). For pure culture isolation, the hyphal tip of a small fungal colony was cut and transferred to PDA plates. Preliminary identification of the collected entomopathogenic fungi was conducted by examining macroand microscopic features of the colonies after culturing fungi on PDA plates at $28^{\circ} \mathrm{C}$. The morpho-taxonomic characteristics of conidia-forming mycelia and conidia structures were identified based on Samson (1974), Humber (1997), and Tasanathai et al. (2010).

Ribosomal RNA genes (rDNA) typically exist as a tandem repeat that includes coding regions, which are conserved to varying degrees, as well as highly divergent spacer regions. These spacer regions, or internal transcribed spacer (ITS) sequences, are widely used in fungal systematics (Driver et al., 2000). In the case of the genus Paecilomyces, analysis of sequences of the large and small subunit rRNA genes indicates the polyphylogeny of the genus (Obornik et al., 2001; Inglis and Tigano, 2006). Therefore, rDNA-ITS sequencing was used to identify Paecilomyces isolates held in our culture collections. A single pair of primers 18S-ITS15.8S-ITS2-28S rDNA was custom-synthesized by Nam Khoa BioTek Company (Ho Chi Minh City, Vietnam). The polymerase chain reaction (PCR) products were checked using agarose gel electrophoresis. DNA sequences were compared to sequences deposited in the National Center of Biotechnology Information (NCBI) server (http://www.ncbinlm.nih.gov) with Entrez and BLAST.

\section{Preparation of spore suspensions}

The sterilized substrate (a mixture of $25 \mathrm{~g}$ rice and $25 \mathrm{~mL}$ distilled water, at $\mathrm{pH}$ 6.5) filled in $250-\mathrm{mL}$ conical flasks was inoculated with $10^{8}$ conidia of fungal isolates. The inoculated medium was incubated in the dark at $28^{\circ} \mathrm{C}$ for 14 days. The fermented substrate $(5 \mathrm{~g})$ was collected in a sterile test tube, supplemented with $10 \mathrm{~mL}$ of $0.05 \%$ Tween 80 , and vortexed for $2 \mathrm{~min}$ to dislodge and suspend the spores before being filtered through a double-layered sterile cheese cloth. The spore concentration was determined using a hemocytometer (Marienfeld GmbH, Marienfeld, Germany) and standardized to $10^{8}$ spores. $\mathrm{mL}^{-1}$ for further uses.

\section{litura}

In vitro pathogenicity of Paecilomyses to $\mathrm{Pl}$. xylostella and $S$.

Third instar larvae of Pl. xylostella and S. litura were obtained from a laboratory colony maintained at $15{ }^{\circ} \mathrm{C}$ and $15: 9 \mathrm{~h}$ light:dark and reared on wheat germ diet until used in this study (Altre et al., 1999). Cultures of isolates were maintained for 25 days at $28{ }^{\circ} \mathrm{C}$ and used for the bioassay. Petri dishes were prepared by placing filter paper, lightly moistened with sterile distilled water at the bottom of the dish. Third instar of $P l$. xylostella and $S$. litura larvae were dipped for 5 seconds in a spore suspension of each isolate $\left(10^{8}\right.$ spores. $\left.\mathrm{mL}^{-1}\right)$ and placed on moistened sterile Whatman no. 4 filter paper (Whatman International Ltd., Maidstone, UK) in a Petri dish. A control 
282

larvae were immersed in sterile aqueous $0.05 \%$ Tween- 80 solutions. For each treatment, ten larvae were kept in dishes with cabbage (Brassica chinensis L var. gracious) as food, and mortality was recorded daily. A corrected mortality and the lethal time causing $50 \%$ mortality $\left(\mathrm{LT}_{50}\right)$ were calculated using the formula recommended by the Food and Agriculture Organization (FAO) of the United Nations (Abbott, 1925; FAO, 2004; Sun et al., 2011). The assay was repeated three times. Corrected mortality $=[$ treated mortality $\%$ - control mortality $\%] /[100$ control mortality \%]. spp.

Effect of the solid substrate on spore production of Paecilomyces

Different concentrations of solid substrates of brown rice (Oryza sativa L.), rough rice (Oryza sativa L.), bran, and rice husks, were used for testing spore production. Each 250-mL conical flask was filled with $25 \mathrm{~g}$ of substrate, mixed with $25 \mathrm{~mL}$ of distilled water, and sterilized in an autoclave at $121{ }^{\circ} \mathrm{C}$ for 20 min. The sterilized substrate was inoculated with $10^{8}$ spores of fungal isolates and incubated in the dark at $28^{\circ} \mathrm{C}$ for 10 days. Five grams of the fermented substrate was collected in a sterile test tube, supplemented with $10 \mathrm{~mL}$ of $0.05 \%$ Tween 80 , and vortexed for $2 \mathrm{~min}$. The spores were suspended before being filtered through double-layered sterile cheesecloth. Spore concentrations were detected using a hemocytometer (Marienfeld GmbH, Marienfeld, Germany), and the number of spores was analyzed to determine the most suitable medium for spore production of Paecilomyces.

\section{Statistical analysis}

Data shown in Tables 1 and 2 and Fig. $2 \mathrm{~A}$ and $2 \mathrm{~B}$ are the means of at least three independent sets of experiments with similar results. Measurements of $\mathrm{LT}_{50}$ values, $\%$ mortality, and the number of spores were analyzed by an analysis of variance (ANOVA) with a completely randomized design. For significant values, means were separated by the least significant difference (LSD) test at $\mathrm{p} \leq 0.05$ using PC SAS 8.2 (SAS Institute, Cary, NC, USA).

\section{Results}

\section{Isolation and identification of Paecilomycesspp.}

Six strains of Paecilomyces isolates, designed P. lilacinus (PL) 01, P. javanicus (PJ) 01, PJ02, PJ03, PJ04, and PJ05 were isolated from the original infected insects. The growth of colonies of PL01 on PDA plates formed a basal felt with floccose aerial mycelium giving rise to conidiophores. The diameter of the colony reached $3.2 \mathrm{~cm}$ within 15 days of incubation at $28{ }^{\circ} \mathrm{C}$. Conidial heads appeared white but gradually became light brownish when sporulated (Fig. 1A). This color change is consistent with species of $P$. lilacinus reported by Samson (1974). The polyphylogeny of the genus Paecilomyces was previously observed by analysis of rRNA gene sequences (Luangsa-ard et al., 2004), and the ITS region was found to be useful in Paecilomyces taxonomy and classification of our isolates. Sequence analysis of the PL01 clone showed $100 \%$ similarity to the $P$. lilacinus sequence in the GenBank database (accession no. AB 1033801.1) and confirmed that the PL01 isolate was the species P. lilacinus.

For other isolates, PJ01 PJ05, colonies grew slowly on PDA with diameters of colonies ranging $2.6 \sim 3.1 \mathrm{~cm}$ within 10 days of incubation at $28{ }^{\circ} \mathrm{C}$. Colonies were round, flat, short floccose, and white, then became cream-colored with age (Fig. 1B). Conidiophores are erect, arising from the aerial mycelium bearing branches with phialides, consisting of a cylindrical basal part, tapering into a thin neck. Conidia are fusiform, sometimes cylindrical, hyaline, and smooth-walled, which is consistent with species of $P$. javanicus reported by Samson (1974). Sequence analysis of the PJ01, PJ02, PJ03, PJ04, and PJ05 clones also showed $100 \%$ similarity to the $P$. javanicus sequence in the GenBank database (accession no. AB 263744.1) and confirmed that PJ01, PJ02, PJ03, PJ04, and PJ05 isolates were the species $P$. javanicus.

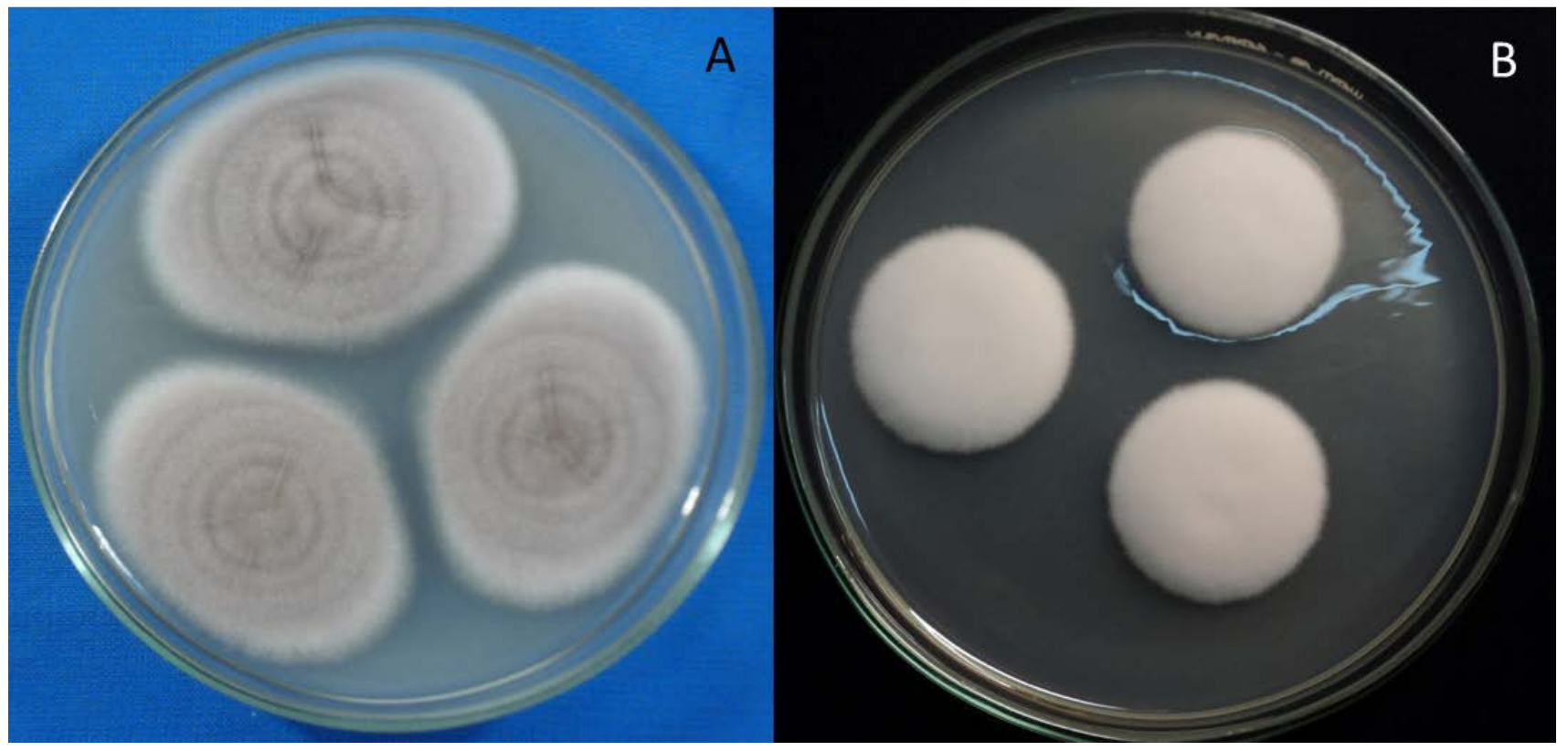

Fig. 1. Colony characteristic of isolated Paecilomyces lilacinus (A) and P. javanicus (B) on PDA medium 

litura

In vitro pathogenicity of Paecilomyces to Pl. xylostella and $S$.

To investigate the pathogenicity of isolates to insects, percentages of corrected mortality for $P$. xylostella third instar larvae exposed to six isolates were calculated. Virulence of the six isolates against $\mathrm{Pl}$. xylostella and $S$. litura significantly differed $(p<0.5)$ (Table 1). In the pathogenicity tests, all fungal isolates were pathogenic to Pl. xylostella and S. litura. The mortality of Pl. xylostella was 100\% with the PL01, PJ02, and PJ04 isolates after 6 days of treatment (Fig. 2A). The $P$. lilacinus isolate strain PL01 showed the highest pathogenicity to Pl. xylostella and $S$. litura with respective $\mathrm{LT}_{50}$ values of 2.51 and 7.09 days. Two P. javanicus isolates, strains PJ04 and PJ02, also strongly infected $P l$. xylostella with respective $\mathrm{LT}_{50}$ values of 2.52 and 2.55 days, while isolates PJ01 and PJ03 demonstrated the lowest pathogenicity to Pl. xylostella (6.56 days) and $S$. litura (17.26 days). Furthermore, six isolates showed higher virulence against $P l$. xylostella than $S$. litura. More than $90 \%$ of Pl. xylostella was infected by four isolates (PL01, PJ02, PJ03, and PJ04) after 6 days of treatment, while all six isolates weakly infected $S$. litura with mortalities below $72 \%$ after 10 days of treatment (Fig. 2B). spp.

Effect of solid substrates on spore production by Paecilomyces

Agro-industrial residues such as brown rice, rough rice, bran, and rice husks, were used as substrates, and spore production of two potential isolates, PL01 and PJ04, was compared. The number of spores varied between the two isolates and among various media (Table 2). The isolated $P$. lilacinus strain, PL01, showed the highest number of spores from brown rice alone as a substrate $\left(32.83 \times 10^{8}\right.$ spores. $\left.^{-1}\right)$ followed by brown rice substrate mixed with rice husks, and wheat bran $\left(13.36 \times 10^{8}\right.$ spores. $\left.^{-1}\right)$ or rice bran $\left(15.67 \times 10^{8}\right.$ spores. $\left.\mathrm{g}^{-1}\right)$. The lowest number of spores was observed from the

corn bran substrate when the PL01 isolate was grown in M5 medium. For the PJ04 isolate, brown rice mixed with wheat bran and rice husks was the most suitable medium for sporulation of the isolated $P$. javanicus with a number of spores of $9.67 \times 10^{8}$ spores..$^{-1}$, while the rough rice substrate alone was unsuitable for cultivating PJ04 to produce spores. Generally, brown rice, wheat bran, and rice bran were suitable for sporulation of both PL01 and PJ04 isolates.
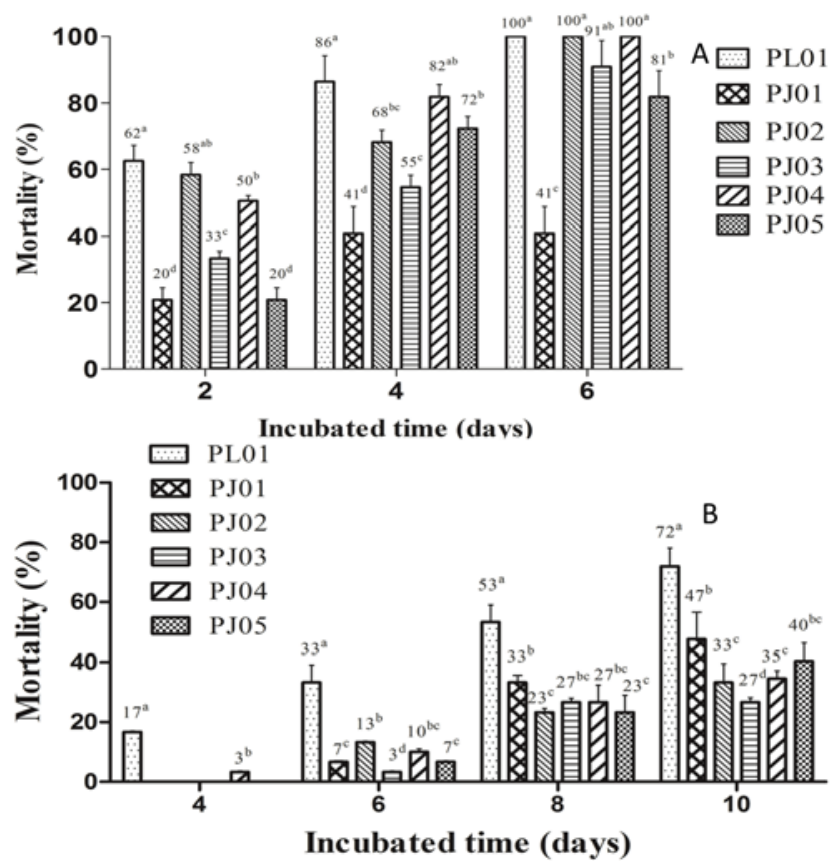

Fig. 2. Corrected mortality of third instar larvae of Plutella xylostella (A) and Spodoptera litura (B) with incubation times (day) when exposed to six isolates

Table 1. Lethal time (days) for $50 \%$ mortality $\left(\mathrm{LT}_{50}\right)$ of Plutella xylostella and Spodoptera litura third instar larvae exposed to six strains of Paecilomyces spp. isolates

\begin{tabular}{|c|c|c|c|}
\hline \multirow{2}{*}{ Isolate } & \multirow{2}{*}{ Fungal species } & \multicolumn{2}{|c|}{$\mathrm{LT}_{50}$ value (days) } \\
\hline & & Plutella xylostella & Spodoptera litura \\
\hline PL01 & P. lilacinus & $2.50^{\mathrm{c}}$ & $7.09^{\mathrm{e}}$ \\
\hline PJ01 & P. javanicus & $6.56^{\mathrm{a}}$ & $10.10^{d}$ \\
\hline PJ02 & P. javanicus & $2.55^{\mathrm{c}}$ & $11.31^{b c}$ \\
\hline PJ03 & P. javanicus & $3.36^{\mathrm{b}}$ & $17.26^{a}$ \\
\hline PJ04 & P. javanicus & $2.52^{\mathrm{c}}$ & $10.53^{\mathrm{cd}}$ \\
\hline PJ05 & P. javanicus & $3.61^{\mathrm{b}}$ & $11.91^{\mathrm{b}}$ \\
\hline
\end{tabular}

Values in the same column with different letters significantly differ $(p<0.05)$.

Table 2. The number of spores of isolated Paecilomyces lilacinus strain PL01 and P. javanicus strain PJ04 on different substrates

\begin{tabular}{|c|c|c|c|c|c|c|c|c|}
\hline \multirow{2}{*}{ Media } & \multicolumn{6}{|c|}{ Composition (\%) } & \multicolumn{2}{|c|}{$\begin{array}{l}\text { Number of spores } \\
\left(\times 10^{8} \text { spores. } \mathrm{g}^{-1}\right)\end{array}$} \\
\hline & Brown rice & Rough rice & Wheat bran & Rice husks & Rice bran & Corn bran & PL01 & PJ04 \\
\hline M1 & 100 & 0 & 0 & 0 & 0 & 0 & $32.83^{a}$ & $4.09^{b}$ \\
\hline M2 & 0 & 100 & 0 & 0 & 0 & 0 & $6.93^{e}$ & $0.55^{\mathrm{e}}$ \\
\hline M3 & 60 & 0 & 30 & 10 & 0 & 0 & $13.36^{b c}$ & $9.67^{\mathrm{a}}$ \\
\hline M4 & 60 & 0 & 0 & 10 & 30 & 0 & $14.67^{b}$ & $4.75^{b}$ \\
\hline M5 & 60 & 0 & 0 & 10 & 0 & 30 & $1.78^{\mathrm{f}}$ & $2.02^{\mathrm{c}}$ \\
\hline M6 & 0 & 70 & 30 & 0 & 0 & 0 & $8.42^{\mathrm{d}}$ & $1.17^{\mathrm{d}}$ \\
\hline M7 & 0 & 70 & 0 & 0 & 30 & 0 & $10.50^{\mathrm{cd}}$ & $4.54^{b}$ \\
\hline M8 & 0 & 70 & 0 & 0 & 0 & 30 & $5.09^{e}$ & $1.00^{\mathrm{d}}$ \\
\hline
\end{tabular}

Values in the same column with different letters significantly differ $(p<0.05)$. 


\section{Discussion}

Entomopathogenic fungi are currently considered to be the important factors controlling insect population, and are well suited for being developed as ecofriendly biopesticide (Kumar et al., 2015). Among them, Peacilomyces are important natural control agents and sources of mycopesticides for pests management worldwide (Han et al., 2014; Jamali and Ghasemi, 2016; Sanjaya et al., 2016). To isolate entomopathogenic fungi, susceptible insect host and selective media have been utilizing to the isolation of entomopathogenic fungi from soil. Use of infected insect is a very sensitive detection method where entomopathogenic fungi can be selectively isolated (Ibrahim et al., 2016). In this study, six strains of Paecilomyces were isolated from infected insects and identified as $P$. lilacinus and $P$. javanicus based on morphological and molecule studies. The macro- and micromorphological features of these fungi grown on PDA agreed with the features reported by Samson (1974) for P. lilacinus and $P$.javanicus. The further studies based on sequence analysis using ITS region confirmed that the PL01 isolate was the species P. lilacinus (accession no. AB 1033801.1) and PJ01, PJ02, PJ03, PJ04, and PJ05 isolates were the species P.javanicus (accession no. AB 263744.1 in the GenBank database).

Several studies have examined the potential use of Paecilomyces as biological control agents. Kepenekci et al. (2015) reported a potential infectivity of $P$. lilacinus isolated in Turkey against the last larvae instar of the potato tuber moth [(Phthorimaea operculella (Zeller)] and the Colorado potato beetle [(Leptinotarsa decemlineata (Say)] (43.3\% and 33.2\% mortality, respectively) on the $10^{\text {th }}$ day of treatment with the fungal concentration of $10^{8} \mathrm{cfu} \cdot \mathrm{mL}^{-1}$. Another study by Lopez et al. (2014) revealed that $P$. lilacinus illustrated the potential pathogenicity against cotton aphids and herbivores under greenhouse and field conditions. In this study, all six isolates showed a strong virulence against the diamondback moth $(P l$. xylostella), with $\mathrm{LT}_{50}$ values ranging $2.5 \sim 6.5$ days. The pathogenicity of the six isolates to $\mathrm{Pl}$. xylostella was much higher than to $S$. litura. Infection with entomopathogenic fungi also differs depending on the ability of the fungal strains to directly penetrate the insect through the cuticle. It seems that it was more difficult to infect $S$. litura than Pl. xylostella by these entomopathogenic fungi. Among the six isolates, $P$. lilacinus showed the highest infectivity against both $S$. litura and $P l$. xylostella. In addition, $P$. lilacinus has been used as an efficient nematicide against nematodes (Meloidogyne spp.) (Sharma et al., 2014; Wang et al., 2010; Sharma et al., 2014). Moreover, $P$. fumosoroseus-infected Pl. xylostella exhibited the highest mortality of $79 \%$ (Altre et al., 1999), and yet P. tenuipes infected $P l$. xylostella third instar larvae with an $\mathrm{LT}_{50}$ of 2.33 days at a spore concentration of $10^{8}$ spores. $\mathrm{mL}^{-1}$ (Baksh and Khan, 2012). In our study, the newly isolated P. lilacinus PL01 and $P$. javanicus PJ04 strains were potential biological control agents and can be used for crop protection against $P l$. xylostella and S. litura.

Paecilomyces isolates were firstly selected for diamondback moth and Oriental leafworm moth control due to the high mortality against the target organism. They are the only pathogens that have been developed for the control of insects with piercing and sucking mouthparts (Eslamizadeh et al., 2015). Entomopathogenic fungi usually infect insect hosts by penetrating the cuticle after their conidia attach and germinate on the insect host cuticle (Han et al., 2014). The synthesis of extracellular enzymes is crucial for the infection process. These fungi secrete extracellular enzymes proteases, chitinases and lipases to degrade the major constituents of the cuticle (protein, chitin and lipids) and allow hyphapenetration (Moorthi et al., 2014; Ibrahim et al., 2016).

Entomopathogenic fungi are an important option in integrated pest management programs so large scale production of the ecofriendly biopesticide is a primary objective in the biocontrol programs for increasing agricultural output. A high spore number is one of the main criteria for choosing a fungal pathogen for biological control of pests in the field. The success of microbial control often depends on producing the pathogen at competitive prices. To achieve lowcosts and high yields of viable fungal spores and make good use of agricultural wastes, the biotechnological potential of these agroindustrial refuse can be employed in byproduct development and establishing improved biocontrol programs. Several studies were performed on utilizing agro-industrial residues as materials for spore production. The highest number of spores in Metarhizium flavoviride was $102.80 \times 10^{9}$ spores. $^{-1}$ sorghum, whereas white-rice yielded the greatest amount of spores for Beauveria bassiana $\left(141.0 \times 10^{9}\right.$ spores. $\left.^{-1}\right)$ after 60 days of incubation (Mar et al., 2012). Robl et al. (2009) reported that refused potatoes yielded the highest amount of spores for $P$. lilacinus $\left(1.75 \times 10^{8}\right.$ spores. $\left.^{-1}\right)$ after 14 days of incubation followed by $80 \%$ cassava bagasse supplemented with $20 \%$ coffee husks $\left(1.56 \times 10^{8}\right.$ spores. $\left.^{-1}\right)$, wheat $\left(1.18 \times 10^{8}\right.$ spores. $\left.g^{-1}\right)$, rye $\left(1.08 \times 10^{8}\right.$ spores. $\left.g^{-1}\right)$, barley $\left(0.80 \times 10^{8}\right.$ spores.g $\left.{ }^{1}\right)$, cassava bagasse $\left(0.69 \times 10^{8}\right.$ spores. $\left.^{-1}\right)$, and soy $\left(0.67 \times 10^{8}\right.$ spores. $\left.g^{-1}\right)$. Kamp and Bidochka (2002) demonstrated that spore production by Beauveria bassiana, Metarbizium anisopliae, and Verticillium lecanii was affected by different solid substrate culture conditions. In our study, mixed substrate fermentation was carried out to make the substrate less expensive and also to test whether they had any beneficial effects on spore production. Methods for commercial production of spores were evaluated on solid substrates consisting of brown rice, rough rice, rice husks, rice bran, wheat bran, and corn bran, and spore production of six isolates was affected by the different solid substrate culture conditions. Different nutrient types in the media resulted in variability in the number of spores produced after the 10-day growth period. Among all the natural media assayed, brown rice alone as a substrate or brown rice mixed with rice husks and wheat bran or rice bran was suitable for the sporulation of both isolated species: $P$. lilacinus and $P$. javanicus. It may because the grains are carbon sources in the form of starch and the utilization of starch of these isolates depends upon its hydrolysis by the action of the enzyme amylase (Mishra and Thawani, 2016). In addition, it was reported that amount of moistening and other agents also played very important role for the growth of fungus (Mishra and Thawani, 2016). High amount of moistening agent caused clumping of the substrate particles, which hindered the optimal utilization of substrate whereas less amount of moistening agent caused dried and non soft condition of the grain and thus fungus could not grow and sporulate. Furthermore, a nutrient-rich medium might not stimulate sporulation, while a nutrient-poor medium would 
not offer extensive mycelial growth. In agreement with study reported by Mishra and Thawani (2016), due to maximal spore production efficiency, rice was suggested as a good solid substrate for the mass production of spores. These tested substrates in our study can be applied by industry to culture two isolates of entomopathogenic fungi for spore production at low costs and with a high concentration of fungal spores.

\section{Conclusions}

The strongly virulent $P$. lilacinus and $P$. javanicus against $P l$. xylostella and S. litura were isolated. Among six isolates, $P$. lilacinus strain PL01 showed the highest pathogenicity to both Pl. xylostella and S. litura with respective $\mathrm{LT}_{50}$ values of 2.51 and 7.09 days. Five isolated $P$. javanicus strains also strongly infected Pl. xylostella with $\mathrm{LT}_{50}$ values of 2.52 6.56 days. For sporulation of these isolates, brown rice alone or mixed with rice husks, wheat bran, or rice bran was suitable for cultivation with low costs and high yields of spores. The newly isolated $P$. lilacinus and $P$. javanicus strains can be used as biological control agents for controlling Pl.xylostella and S. litura.

\section{References}

Abbott WS (1925). A method of computing the effectiveness of an insecticide.Journal of Economic Entomology 18:265-267.

Altre JA, Vandenberg JD, Cantone FA (1999). Pathogenicity of Paecilomyces fumosoroseus isolates to diamondback moth, Plutella xylostella: correlation with spore size, germination speed, and attachment tocuticle.Journal of Invertebrate Pathology 73:332-338.

Baksh A, Khan A (2012). Pathogenicity of Paecilomyces tenuipes to diamondback moth, Plutella xylostella at three temperatures in Trinidad. International Journal of Agriculture and Biology 14:261-265.

Buzzini P, Martini A (1999). Production of carotenoids by strains of Rhodotorula glutinis culturedin raw materials of agro-industrial origin. Bioresource Technology 71:41-44.

Cliquet S, Jackson MA (2005). Impact of carbon and nitrogen nutrition on the quality, yield and composition of blastospores of the bioinsecticidal fungus Paecilomyces fumosoroseus. Journal of Industrial Microbiology and Biotechnology 32:204210.

Driver F, Milner RJ, Trueman JWH (2000). A taxonomic revision of Metarhizizum based on a phylogenetic analysis of rDNA sequence data. Mycological Research 104:143-150.

Eslamizadeh R, Sajap ASB, Omar DB, Adam NAB (2015). Evaluation of different isolates of entomopathogenic fungus, Paecilomyces fumosoroseus (Deuteromycotina: Hyphomycetes) against Bemisia tabaci (Hemiptera: Aleyrodidae). Biological Control in Plant Protection 2:82-90.

FAO (Food and Agriculture Organization of the United Nations) (2004). Resistance management and integrated parasites control in ruminants/guidelines. In: module 1-ticks: acaricide resistance, diagnosis, management and prevention. Food and Agriculture Organization, Animal Production and Health Division, Rome, 25-77.

Fiedler Ż, Sosnowska D (2007). Nematophagous fungus Paecilomyces lilacinus (Thom) Samson is also a biological agent for control of greenhouse insects and mite pests. BioControl 52:547-558.
Gao L, Sun MH, Liu XZ, Che YS (2007). Effects of carbon concentration and carbon to nitrogen ratio on the growth and sporulation of several biocontrol fungi. Mycological Research 111:87-92.

Han JH, Jin BR, Kim JJ, Lee SY (2014). Virulence of entomopathogenic fungi Metarbizium anisopliae and Paecilomyces fumosoroseus for the microbial control of Spodoptera exigua. Mycobiology 42:385-390.

Humber RA (1997). Fungi: identification. In: Lacey LA (Ed). Manual of techniques in insect pathology. Academic Press, San Diego, USA pp 153-185.

Ibrahim AA, Mohamed HF, El-Naggar SEM, Swelim MA, Elkhawaga OE (2016). Isolation and selection of entomopathogenic fungi as biocontrol agent against the greater wax moth, Galleria mellonella L.(Lepidoptera: Pyralidae).Egyptian Journal of Biological Pest Control 26:249-253

Inglis PW, Tigano MS (2006). Identification and taxonomy of some entomopathogenic Paecilomyces spp. (Ascomycota) isolates using rDNA-ITS Sequences. Genetics and Molecular Biology 29:132-136.

Jamali S, Ghasemi F (2016). Pathogenicity of Paecilomyces marquandii on eggs of Meloidogyne incognita. Journal ofCrop Protection 5:125-130.

Kamp AM, Bidochka MJ, (2002). Conidium production by insect pathogenic fungi on commercially available agars. Letters in Applied Microbiology 35:7477.

Kepenekci I, Oksal E, Saglam HD, Atay T, Tulek A, Evlice E (2015). Identification of Turkish isolate of the entomopathogenic fungi, Purpureocillium lilacinum (syn: Paecilomyces lilacinus) and its effect on potato pests, Phthorimaea operculella (Zeller)(Lepidoptera: Gelechiidae) and Leptinotarsa decemlineata (Say)(Coleoptera: Chrysomelidae). Egyptian Journal of Biological Pest Control 25:121-127.

Kerry BR (1990). An assessment of progress toward microbial control of plant-parasitic nematodes. Journal of Nematology.22:621.

KiewnickS, Sikora RA (2006). Biological control of the root-knot nematode Meloidogyne incognita by Paecilomyces lilacinus strain 251. Biological Control 38:179-187.

Kumar CS, Jacob TK, Devasahayam S, D'Silva S, Kumar NK (2015). Isolation and characterization of a Lecanicillium psalliotae isolate infecting cardamom thrips (Sciothrips cardamomi) in India. BioControl 60:363-373.

Lacey LA, Fransen JJ, Carruthers R(1996). Global distribution of a naturally occurring fungi of Bemisia, their biologies and use as biological control agents. In: Gerling D, Mayer R(Eds). Bemisia: 1995, taxonomy, biology, damage, control and management. Intercept, Andover, Hants, UK pp 401433.

Lane BS, Trinci AP, Gillespie AT (1991). Influence of cultural conditions on the virulence of conidia and blastospores of Beawveria bassiana to the green leafhopper, Nephotettix virescens. Mycological Research 95:829833.

Lopez DC, Zhu-Salzman K, Ek-Ramos MJ, Sword GA (2014). The entomopathogenic fungal endophytes Purpureocillium lilacinum (formerly Paecilomyces lilacinus) and Beawveria bassiana negatively affect cotton aphid reproduction under both greenhouse and field conditions. PloSone 9:p.e103891.

Luangsa-ard JJ, Hywel-Jones NL, Samson RA (2004). The polyphyletic nature of Paecilomyces sensu lato based on 18S-generated rDNA phylogeny. Mycologia 96:773-780. 
286

Mar TT, Suwannarach N, Lumyong S (2012). Isolation of entomopathogenic fungi from Northern Thailand and their production in cereal grains. World Journal of Microbiology and Biotechnology 28:3281-3291.

Mishra PK, Thawani V (2016). Mass production of Paecilomyces Fumosoroseus from agricultural products and waste material. PARIPEXIndian Journal of Research 5:271-273.

Moorthi V, Balasubramanian PC, Avery PB, Selvarani S, Kubendran T, Rathinakumar T, Banu AN (2014). Pathogenicity and proteome production of Isaria fumosorosea (Paecilomyces Fumosoroseus) (WISE) isolates against lemon butterfly, Papilio demoleus (Papilionidae: lepidoptera). African Journal of Biotechnology 13:4176-4182.

Obornik M, Jirku M, Dolezel D (2001). Phylogeny of mitosporic entomopathogenic fungi: Is the genus Paecilomyces polyphyletic? Canadian Journal of Microbiology 47:813-819.

Pandey A, Soccol CR, Nigam P, Soccol VT (2000). Biotechnological potential of agro-industrial residues. I: sugarcane bagasse. Bioresource Technology 74:69-80.

Pau CG, Leong S, Teck C, Wong SK, Eng L, Jiwan M, ... Majid NM (2012). Isolation of indigenous strains of Paecilomyces lilacinus with antagonistic activity against Meloidogyne incognita. International Journal of Agriculture and Biology 14:197-202.

Perez CJ, Shelton AM (1997). Resistance of Plutella xylostella (Lepidoptera: Plutellidae) to Bacillus thuringiensis Berliner in Central America. Journal of Economic Entomology 90:87-93.

Robl D, Sung LB, Novakovich JH, Marangoni PR, Zawadneak MAC, Dalzoto PR, ... Pimentel IC (2009). Spore production in Paecilomyces lilacinus (Thom.) Samson strains on agro-industrial residues. Brazilian Journal of Microbiology 40:296-300.

Samson RA (1974). Paecilomyces and some allied hyphomycetes. Studies in Mycology 6:1-119.

Sanjaya Y, Ocampo VR, Caoili BL (2016). Pathogenicity of three entomopathogenic fungi, Metarhizium anisopliae, Beawveria bassiana, and Paecilomyces lilacinus, to Tetranychus kanzawai infesting papaya seedlings. Arthropods 5:109-113.

Santa HSD, Santa ORD, Brand D, Vandenberghe LPDS, Soccol CR (2005). Spore production of Beawveria bassiana from agro-industrial residues. Brazilian Archives of Biology and Technology 48:51-60.

Sharma A, Sharma S, Dalela M (2014). Nematicidal activity of Paecilomyces lilacinus 6029 cultured on Karanja cake medium. Microbial Pathogenesis 75:16-20.

Shelton AM, Wyman JA (1992). Insecticide resistance of diamondback moth in North America. In: Talekar NS (Ed). Diamondback moth and other crucifer pests. Proceedings of the Second International Workshop, Tainan, Taiwan.
SiddiquiZA, Futai K (2009). Biocontrol of Meloidogyne incognita on tomato using antagonistic fungi, plant-growth-promoting rhizobacteria and cattle manure. Pest Management Science 65:943-948.

Soccol CR, Vandenberghe LPS (2003). Overview of applied solid-state fermentation in Brazil. Biochemical Engineering Journal 13:205-218.

Sun M, Ren Q, Guan G, Liu Z, Ma M, Gou H, ... YangJ (2011). Virulence of Beawveria bassiana, Metarbizium anisopliae and Paecilomyces lilacinus to the engorged female Hyalomma anatolicum anatolicum tick (Acari: Ixodidae). Veterinary Parasitology 180:389-393.

Syed AR (1992). Insecticide resistance in diamondback moth in Malaysia. In: Talekar NS (Ed). Management of diamondback moth and other crucifer pests. Proceedings of the Second International workshop, Asia Vegetable Research and Development Center, Taiwan, pp 437-442.

Tabashnik BE, Cushing NL, Finson N, Johnson MW (1990). Field development of resistance to Bacillus thuringiensis in diamondback moth (Lepidoptera: Plutellidae). Journal of Economic Entomology 83:1671-1676.

Talekar NS, Shelton AM (1993). Biology, ecology, and management of the diamondback moth. Annual Review of Entomology 38:275-301.

Tasanathai K, Luangsa-ard JJ, Mongkolsamrit S, Hywel-Jones NL (2010). Atlas of invertebrate-pathogenic fungi of Thailand. Thailand, National Center for Genetic Engineering and Biotechnology.

Wang J, Wang J, Liu F, Pan C (2010). Enhancing the virulence of Paecilomyces lilacinus against Meloidogyne incognita eggs by overexpression of a serine protease. Biotechnology Letters 32:11591166.

Wraight SP, Carruthers R, Bradley CA, Jaronski ST, Lacey LA, Wood P, Galaini-Wraight S (1998). Pathogenicity of the entomopathogenic fungi Paecilomyces spp. and Beawveria bassiana against the silverleat whitefly, Bemisia argentifolii. Journal of Invertebrate Pathology 71:217226.

Wraight SP, Carruthers RI, Jaronski ST, Bradley CA, Garza CJ, GalainiWraightS (2000). Evaluation of the entomopathogenic fungi Beawveria bassiana and Paecilomyces fumosoroseus for microbial control of the silverleaf whitefly, Bemisia argentifolii. Biological Control 17:203-217.

Ying SH, Feng MG (2006). Medium components and culture conditions affect the thermotolerance of aerial conidia of fungal biocontrol agent Beauveria bassiana. Letters in Applied Microbiology 43:331-335.

Zawadneak MAC, Pimentel IC, Robl D, Dalzoto P, Vicente V, SosaGómez DR, ... Cuquel FL (2015). Paecilomyces niveus Stolk \& Samson, 1971 (Ascomycota: Thermoascaceae) as a pathogen of Nasonovia ribisnigri (Mosley, 1841) (Hemiptera, Aphididae) in Brazil. Brazilian Journal of Biology 75:158-162. 